\title{
Análisis de escenarios de cambio climático A1B, A2 y B1 para la reserva forestal protectora bosque oriental de Bogotá años 2040, 2070 y 2095 utilizando MarksimGCM'1
}

\author{
Analysis of climate change scenarios A1B, A2 and B1 for \\ protective forest reserve Bosque oriental de Bogotá, \\ years 2040, 2070 \& 2095 with MarksimGCM
}

Análise da mudança climática cenários A1B, A2 e B1 para proteção reserva florestal do leste da floresta Bogotá anos 2040, 2070 e 2095 usando MarksimGCM

Fecha de recepción: mayo de 2013

Fecha de aprobación: agosto de 2013
Johny Roberto Rodríguez Pérez ${ }^{2}$ Julio Eduardo Beltrán Vargas ${ }^{3}$

\section{Resumen}

El siguiente artículo presenta los resultados y análisis de las condiciones climáticas futuras de temperatura y precipitación para la Reserva Forestal Bosque Protector Cerros Orientales de Bogotá (RFPBOB). Para esto, se simularon los escenarios de cambio climático: A1B, A2 y B1 en los años 2040, 2070 y 2095. La simulación de las condiciones climáticas futuras en la RFPBOB tuvo en cuenta las variables: temperatura y precipitación, descritas bajo escenarios de cambio climático aplicando modelos de circulación general en MarkSimGCM. Se encontró que para todos los escenarios la temperatura aumentará para el año 2095, respecto al periodo de referencia 1960-1990. Los incrementos esperados para cada escenario son: 3,6 ${ }^{\circ} \mathrm{C} \mathrm{A} 1 \mathrm{~B}, 4,3{ }^{\circ} \mathrm{C} \mathrm{A} 2,2,5^{\circ} \mathrm{C} \mathrm{B} 1$. El escenario más caluroso es el $\mathrm{A} 2$, seguido por el A1B y el B1. Para el caso de la precipitación anual la tendencia es hacia el aumento respecto al periodo de referencia en los escenarios A1B y A2, en el escenario B1 la variación no es significativa, 21\% A1B, 14,5\% A2, 1 \% B1. Producto de la comparación de los resultados encontrados en MarkSimGCM con resultados de estudios previos particularmente realizados por el Instituto de Investigaciones Ambientales (Ideam), se encontraron diferencias particularmente en la precipitación, siendo mucho más bajos los incrementos en la simulación efectuada en esta investigación. Para la temperatura, los escenarios realizados por el Ideam coinciden con los discutidos en este estudio. Los escenarios comparados fueron el A1B y el A2.

Palabras clave: Bogotá, cambio climático, escenarios, reserva forestal, simulación.

1 Artículo dei investigación. Producto del trabajo del grupo de investigación Indesos, de la maestría en Desarrollo Sustentable y Gestión Ambiental.

2 Universidad Distrital Francisco José De Caldas, Facultad de Medio Ambiente y Recursos Naturales. Bogotá, Colombia. Contacto: rodrijohny@gmail.com; jonhy.rodriguez@unad.edu.co.

3 Universidad Distrital Francisco José De Caldas, Facultad de Medio Ambiente y Recursos Naturales. Contacto: juliobeltranvargas@gmail.com; jebeltran@udistrital.edu.co. 


\section{Abstract}

The following paper presents the results and analysis of future climate conditions of temperature and precipitation for Reserva Forestal Bosque Protector Cerros Orientales de Bogotá (RFPBOB). In this investigation were simulated climate change scenarios: $\mathrm{A} 1 \mathrm{~B}, \mathrm{~A} 2$ and $\mathrm{B} 1$ in the years 2040, 2070 and 2095. The simulation of future climate conditions in the RFPBOB took into account variables: temperature and precipitation, as described under climate change scenarios using general circulation models on MarkSimGCM. It was found that for all scenarios the temperature increase for the year 2095 compared to the reference period 19601990. The increases are expected for each scenario: $3.6^{\circ} \mathrm{C}, \mathrm{A} 1 \mathrm{~B} ; \mathrm{A} 2,4.3$ ${ }^{\circ} \mathrm{C} ; 2.5^{\circ} \mathrm{C}, \mathrm{B} 1$. The hottest scenario was the $\mathrm{A} 2$, followed by $\mathrm{A} 1 \mathrm{~B}$ and $\mathrm{B} 1$. In the case of the annual precipitation trend was towards the increase over the reference period in scenarios $\mathrm{A} 1 \mathrm{~B}$ and $\mathrm{A} 2, \mathrm{~B} 1$ onstage variation is not significant, $21 \%, \mathrm{~A} 1 \mathrm{~B} ; \mathrm{A} 2,14.5 \% ; 1 \%, \mathrm{~B} 1$. Product of comparison the results from MarkSimGCM with others studies particularly made by the Instituto de Investigaciones Ambientales de Colombia (Ideam) were found differences particularly in precipitation, with much lower increases in the simulation carried out in this research. The scenarios simulated by Ideam match those discussed in this study. The scenarios compared were the $\mathrm{A} 1 \mathrm{~B}$ and $\mathrm{A} 2$.

Keywords: Bogot, climate Cchange, scenarios, forest reserve, simulation.

\section{Resumo}

O artigo a seguir apresenta os resultados e análises das condições climáticas futuras de temperatura e precipitação para a Protecção Forest Hills Reserva Florestal leste de Bogotá (RFPBOB). Para este cenários simulados mudanças climáticas: A1B, A2 e B1, nos anos de 2040, 2070 e 2095. A simulação de condições climáticas futuras na RFPBOB levou em conta variáveis: temperatura e precipitação, conforme descrito em cenários de mudanças climáticas utilizando modelos de circulação geral MarkSimGCM. Verificou-se que em todos os cenários do aumento de temperatura para o ano de 2095 em comparação com o período de referência de 1960-1990. Os aumentos são esperados para cada cenário: $3,6^{\circ} \mathrm{C} \mathrm{A1B}$, A2 $4,3^{\circ} \mathrm{C}, 2,5^{\circ} \mathrm{C} \mathrm{B} 1$. A cena mais quente é o A2, seguido por A1B e B1. No caso de a tendência para a precipitação anual é o aumento no período de referência em cenários $\mathrm{A} 1 \mathrm{~B}$ e A2, B1 palco variação não é significativa, $21 \%$ A1B, A2 14,5\%, 1\% B1. Comparação de produtos MarkSimGCM os resultados encontrados em estudos anteriores, com especial realizado pelo Instituto de Pesquisa Ambiental (diferenças Ideam) foram encontrados principalmente na precipitação, com aumentos muito mais baixos na simulação realizadas nesta pesquisa. Para cenários de temperatura realizadas por Ideam correspondência discutido neste estudo. Os cenários foram comparados a A1B e A2.

Palavras chave: Bogotá, alterações climáticas, cenários, reserva florestal, simulação. 


\section{Introducción}

La actual distribución mundial de la flora refleja una complicada historia de factores interactuantes, el clima es uno de ellos (Millar et al., 2007). El Panel Intergubernamental de Expertos sobre el Cambio Climático llegó a la conclusión de que el calentamiento del clima es un hecho inequívoco y que es, con toda probabilidad, por causa del aumento observado de la concentración de gases de efecto invernadero de origen antrópico en la atmósfera (IPCC, 2007). Además del alza de las temperaturas mundiales promedio, se han constatado cambios apreciables en las temperaturas diurnas, nocturnas y estacionales; la frecuencia, duración e intensidad de las olas de calor, sequías e inundaciones; las pautas de los vientos y tempestades; la cubierta de escarcha, nieve y hielo, y el nivel de los mares (Bernier y Shoene, 2009). El cambio climático como fenómeno de impacto global y probablemente dominado por las respuestas a factores antropogénicos (Johns, et al., 2003) muestra efectos directos en las funciones de los ecosistemas, entendidas estas como procesos físicos, químicos y biológicos que ocurren en los mismos y son necesarias para su automantenimiento (Turner y Chapin, 2005) a su vez, son resultado de interacciones entre componentes bióticos y abióticos de un ecosistema (Groot y Wilson, 2002). Las funciones de los ecosistemas se relacionan directamente con los servicios ambientales a través de los cuales se dan las condiciones y procesos por los cuales los ecosistemas y las especies que los habitan se sostienen y mantienen la vida humana. Los servicios y bienes ecosistémicos son definidos por su contribución al ser humano, puesto que son productos de las diferentes funciones de los ecosistemas, los seres humanos los disfrutan, consumen o utilizan (Dobbs et al., 2011). Desde siempre, la humanidad ha dependido de los servicios que prestan la biosfera y sus ecosistemas (Montes y Sala, 2007); así pues, la alteración de los ecosistemas por el cambio en el clima repercute directamente en los bienes y servicios dados por estos y, por consiguiente, en la calidad de vida de los humanos; la distribución de los ecosistemas está en gran medida determinada por el factor climático, los ecosistemas actuales y futuros son reflejo de una compleja interacción entre las variables biofísicas, como el suelo, clima y uso de la tierra (Chapin et al., 2000; McKenzie et al., 2003; Nitschke e Innes, 2008).
El cambio climático, fenómeno definido como cualquier cambio a largo plazo en el comportamiento del clima en periodos dentro del rango de decadas a millones de años (Pabón, 2006), tiene multiples efectos que intentan ser anticipados. En este sentido, las actuales aproximaciones sobre la determinación de los efectos presentes y futuros del cambio climático se han dado desde el análisis de modelos simulados a diversas escalas, que combinan climas estimados (modelos de circulación general, MCG) con procesos basados en modelos ecofisiológicos y biogeoquímicos (Batchelet et al., 2001). La mayor parte de los escenarios más aceptados provienen de la modelación del clima que utiliza como principal insumo los modelos de circulación general (MCG), también conocidos por su sigla en inglés GCM (Mahlman, 1997). Estos, a su vez, usan como principal insumo la estimación de las futuras emisiones de gases efecto invernadero (Magaña et al., 2000). Así, se ha generado una exigencia cada vez más urgente por desarrollar modelos y escenarios de cambio climático que puedan evaluar aspectos económicos, consecuencias ecológicas y sociales producto de efectos derivados de los cambios (Watson et al., 1996). Sin embargo, la carga de incertidumbre permanece latente en estos modelos, debido a la gran cantidad de variables que deben ser tenidas en cuenta (Mahlman, 1997, Millar et al., 2007). Producto de los modelos, se generan escenarios para escalas regionales o locales, con los cuales se desarrollan criterios desde la gestión ambiental que permiten proponer opciones de adaptación y mitigación ante el cambio futuro (Millar, et al., 2007). Aunque se ha señalado que ninguno de los escenarios se puede suponer más probable que los demás (Castro et al., 2005), las proyecciones de clima utilizando modelos MCG y variantes de este coinciden en dos escenarios posibles denominados A2 y B2 (IPCC, 2001), en los que se da un incremento de temperatura, siendo mayor la elevación para el primero. Estos escenarios indican, con un elevado grado de confianza, que un futuro cambio climático estará marcado por un calentamiento global condicionado por el incremento de emisiones antropogénicas (Castro, et al., 2005). Sin embargo, los modelos MCG deben ser ajustados a las áreas de estudio a través de métodos de downscaling que permiten refinar los análisis 
utilizando procesos dinámicos o estadísticos. Una forma sencilla de hacer esto es utilizando métodos estadísticos en los que, a través de cálculos probabilísticos, se puede inferir sobre las condiciones climáticas futuras; en la actualidad, aplicaciones como MarkSimGCM se utilizan para generar escenarios de cambio climático, este en particular utiliza dos tipos de downscaling para aumentar la resolución de los datos, uno de tipo estocástico y estadístico en donde se proyectan datos utilizando datos presentes (Jones et al., 2009).

La Reserva Forestal Protectora Bosque Oriental de Bogotá (RFPBOB) se localiza sobre los cerros orientales de Bogotá, hace parte del sistema orográfico y de la estructura ecológica principal de la capital. Su ubicación facilita la conexión regional con los parques nacionales naturales de Chingaza y Sumapaz (Carrizosa, 2007). Cortés, Van der Hammen y Rangel (1999) afirman que los bosques montañosos del norte de los Andes están en inminente peligro de desaparición, especialmente los de la cordillera oriental colombiana. La región de la denominada sabana de Bogotá, donde se encuentra la RFPBOB, ha perdido casi la totalidad de la vegetación boscosa primaria, solamente se encuentran pequeños restos de bosques secundarios que han sufrido entresacas y otras manifestaciones antropogénicas.

La RFPBOB hace parte de la estructura ecológica principal de la ciudad de Bogotá, de esta dependen importantes bienes y servicios ecosistémicos: espacio público verde - recreación pasiva y el rencuentro con los valores de la naturaleza, educación e investigación-, regulación climática y ambiental -regulación del riesgo ambiental: deslizamientos, inundaciones, contaminación y ruido, mitigación del cambio climático- y biodiversidad y vida silvestre - especies y ecosistemas como soporte de procesos de vida, disponibles para la investigación, educación, recreación y turismo especializado(Andrade et al., 2008, Sanchez, 2007, Wiesner, 2006). Los cerros soportan las actividades de las comunidades asentadas en el área de la reserva, así como de los ciudadanos de Bogotá (Guerrero, 2006). Los cerros orientales de Bogotá son reconocidos por medio de valores ambientales, económicos y estéticos, a través de categorías que engloban a ecosistemas, especies, suelo y paisaje. De diversas maneras, los cerros se han convertido en elementos centrales del patrimonio ambiental y paisajístico de la región capital; sin embargo, la apropiación histórica del suelo de los cerros ha sido contraria a la vocación del mismo, lo cual ha llevado a una alteración de la estructura ecológica y con esto se ha perturbado la calidad y cantidad de bienes y servicios ecosistémicos (Wiesner, 2006).

Para el Instituto Colombiano de Estudios Ambientales, el cambio climático será más marcado dependiendo de cómo avance el siglo XXI e impactará de forma diversa a los diferentes países del mundo, particularmente a Colombia (Ideam, 2010). Al revisar los estudios relacionados con la modelación del clima para Colombia y la simulación de las condiciones futuras, se encuentran numerosos acercamientos teóricos realizados particularmente en la última década; sin embargo, los escenarios obtenidos no han permitido proyectar las áreas a nivel de paisaje, es por ello que en los estudios adelantados se ha tratado de corregir esta situación utilizando diversos modelos que emplean diferentes tipos de downscaling (Ruiz, 2010).

En esta investigación se realizó el análisis de las variables climáticas temperatura y precipitación bajo escenarios de cambio climático, se presentan los resultados y análisis de la simulación del comportamiento de estas variables climáticas en los escenarios de cambio climático A1B, A2 y B1 utilizando la aplicación MarkSimGCM que se apoya en los modelos de circulación general: CNRM-CM3 (Centre National de Recherches Météorologiques, France), CSIRO-Mk3.5 (Commonwealth Scientific And Industrial Research Organisation Atmospheric Research, Australia), ECHam5 (Max Planck Institute for Meteorology, Germany) y MIROC3.2 (Center for Climate System Research, National Institute for enviromental studies, Frontier Research Center for Global Change, University of Tokyo, Japan) y en datos de la base climática WorldClim, los años estudiados fueron 2040, 2070 y 2095, años utilizados por otros estudios de cambio climático para la zona andina colombiana. Entre los resultados más destacados se encontró que, para todos los escenarios analizados, la temperatura aumentará en mayor o menor grado a lo largo del siglo XXI. El 
escenario más caluroso es el A2, seguido del A1B y el B1. Para la precipitación, la tendencia es hacia el aumento en todos los casos, presentándose mayores precipitaciones en el escenario A1B, seguido del A2 y el B1. Al compararse estos escenarios con los resultados de estudios previos, particularmente los realizados por el Ideam, se encontraron diferencias significativas entre ambos, especialmente en la precipitación, siendo mucho más bajos los incrementos en los resultados estimados por MarkSimGCM que los discutidos en otras investigaciones. Para la temperatura, los resultados obtenidos en los escenarios realizados por el Ideam fueron cercanos a los discutidos en este trabajo. Los escenarios comparados fueron los A1B y A2. Los resultados del escenario B1 obtenidos con MarkSimGCM no se confrontaron ya que no se accedieron a datos comparables.

\section{Metodología}

Para la obtención de la información se tomaron estaciones climatológicas cercanas o dentro de la RFPBOB con el propósito de obtener datos históricos sobre el comportamiento de las variables climáticas temperatura y precipitación que pudieran ser contrastados frente a la línea base trabajada por MarkSimGCM. Una vez determinadas las estaciones climatológicas representativas, se utilizaron las respectivas coordenadas para alimentar a la aplicación MarkSimGCM. Sobre esta, se seleccionaron los modelos de circulación general, los escenarios y los años futuros por trabajar, se obtuvieron datos diarios de precipitación y temperatura por cada estación climatológica. Los datos se promediaron y se contrastaron para determinar diferencias entre años y periodo de referencia.

\section{Obtención de la información}

Se analizó la información climática simulada por MarksimGCM. Esta herramienta maneja como variables de entrada coordenadas geográficas, escenario por analizar y tipo de modelo de circulación general. Se utilizaron como referencia las coordenadas geográficas de cuatro estaciones climatológicas pertenecientes a la ciudad de Bogotá y una estación localizada en el municipio de Choachi (Cundinamarca), (ver tabla 1). La selección de estaciones se realizó bajo dos criterios: disponibilidad de datos actuales y distancia respecto a la RFPBOB.

\begin{tabular}{|c|c|c|c|c|c|c|}
\hline Estación & Municipio & $\begin{array}{l}\text { Fecha } \\
\text { Instalación }\end{array}$ & $\begin{array}{l}\text { Sector representativo } \\
\text { para la RFPBOB }\end{array}$ & $\begin{array}{l}\text { Altitud } \\
\text { (msnm) }\end{array}$ & $\begin{array}{l}\text { Latitud } \\
\text { (N) }\end{array}$ & $\begin{array}{l}\text { Longitud } \\
\text { (W) }\end{array}$ \\
\hline Venado de Oro Vivero & Bogotá & 1965-Ago & Central & 2725 & $4035^{\circ}$ & $74^{\circ} 03^{`}$ \\
\hline Los Taques & Bogotá & 1986-Ene & Sur & 3150 & $4^{\circ} 11^{\prime}$ & $74^{\circ} 11^{\prime}$ \\
\hline La Bolsa & Choachi & 1987-May & Este & 3195 & 4034 & $73^{\circ} 58^{\prime}$ \\
\hline Universidad Nacional & Bogotá & 1987-May & Oeste & 2556 & $4038^{\prime}$ & $74^{\circ} 05^{\circ}$ \\
\hline $\begin{array}{l}\text { Universidad de Ciencias } \\
\text { Aplicadas y Ambientales-UDCA }\end{array}$ & Bogotá & 1988-Dic & Norte & 2570 & $4047^{\prime}$ & $74^{\circ} 02^{`}$ \\
\hline
\end{tabular}

Tabla 1. Ubicación de las estaciones meteorológicas

Fuente: elaboración propia

\section{Simulación de escenarios de cambio climático utilizando MarkSimGCM}

MarkSimGCM es un generador de condiciones climáticas de tercer orden (Jones y Thornton, 1993), que permite simular condiciones climáticas futuras usando modelos de circulación general, y se encuentra disponible en http:/gismap.ciat.cgiar. org/MarkSimGCM

La aplicación cuenta con varios modelos de circulación general: BCCR_BCM2.0, CNRM-CM3, CSIRO-Mk3, 5, ECHam5, INMCM3.0, MIROC3.2, para los escenarios A1B, A2 y B1 del IPCC, los 
cuales pueden ser trabajados individualmente o en conjunto. Para esto, se toman como referencia los datos de la base climática mundial WorldClim, en la cual se reúnen el conjunto de capas del clima global - redes climáticas - con una resolución espacial de 1 kilómetro cuadrado aproximadamente (Hijmans et al., 2005). Para la obtención de datos en el generador se introdujeron, como puntos de análisis, las coordenadas geográficas de las estaciones climáticas de la tabla 1. Se utilizó el valor medio de los modelos de circulación general: CNRM-CM3, CSIRO-Mk3, 5, ECHam5, MIROC3.2 y se utilizó el ensamble de estos modelos dado por la aplicación. Los escenarios de cambio climático estudiados fueron los que permitía el generador, esto es A1B, A2 y B1. Se tomaron tres años para estimar las condiciones de clima futuro de la RFPBOB: 2040, 2070 y 2095. Los cuales corresponden a los últimos o primeros años de los rangos de analizados en los estudios de Ruiz (2010) y (2011) sobre escenarios de cambio climático para Colombia y la región capital.
La aplicación genera datos diarios por año de precipitación y temperatura una vez establecidas las variables de trabajo. Estos datos se promediaron para obtener montos mensuales para los casos de la precipitación promedio mensual y la temperatura promedios mensuales y anuales. La precipitación anual se obtuvo al sumar los valores diarios de precipitación por año. Con los datos obtenidos para los años 2040, 2070 y 2095 se realizó una comparación frente al valor de referencia o línea base dada también por el simulador. Este valor está referido al año 1975, el cual se toma como valor de la línea base ya que es el año medio de los 30 años que conforman el periodo de referencia 1960 a 1990 de los datos climáticos disponibles en Worldclim. Los escenarios utilizados fueron: A1B, A2 y B1, cada uno presenta un mundo bajo condiciones de desarrollo particular (tabla 2). Finalmente, con los datos procesados por escenario, año y estación, fue posible construir isotermas e isoyetas para representar espacialmente los cambios a través del tiempo en la precipitación media y temperatura media anual en la RFPBOB.

\begin{tabular}{|c|c|c|}
\hline Escenario A1B & Escenario A2 & Escenario B1 \\
\hline $\begin{array}{l}\text { El escenario A1B pertenece a la familia de } \\
\text { escenarios A1, que se caracterizan por el } \\
\text { desarrollo alternativo de tecnologías de ener- } \\
\text { gía. De esta familia se desprenden tres tipos } \\
\text { de escenarios: el A1FI caracterizado por la } \\
\text { utilización intensiva de combustibles de ori- } \\
\text { gen fósil, el A1B (equilibrado) y A1T en el que } \\
\text { se da predominantemente el uso de com- } \\
\text { bustibles de origen no fósil. El escenario A1B } \\
\text { además de presentar un futuro equilibrado } \\
\text { entre fuentes de energía supone un com- } \\
\text { portamiento "armonizado"del crecimiento } \\
\text { de la población mundial, el producto interno } \\
\text { bruto y el gasto energético del planeta (IPCC, } \\
\text { 2007). }\end{array}$ & $\begin{array}{l}\text { El escenario A2 describe un } \\
\text { mundo muy heterogéneo. Las } \\
\text { condiciones de este escenario } \\
\text { plantean una población mun- } \\
\text { dial en continuo crecimiento, } \\
\text { un desarrollo económico orien- } \\
\text { tado básicamente a las regio- } \\
\text { nes, y un cambio tecnológico } \\
\text { más fragmentado y lento que } \\
\text { en otras líneas evolutivas de } \\
\text { escenarios de cambio climático } \\
\text { (IPCC, 2007). }\end{array}$ & $\begin{array}{l}\text { De acuerdo con el IPPC (2007) el escenario B1 } \\
\text { describe un mundo con una población mun- } \\
\text { dial que alcanza un máximo hacia mediados } \\
\text { del siglo y desciende posteriormente como en } \\
\text { la línea evolutiva A1, pero con rápidos cambios } \\
\text { de las estructuras económicas orientados a una } \\
\text { economía de servicios y de información, acom- } \\
\text { pañados de una utilización menos intensiva de } \\
\text { materiales y de la introducción de tecnologías } \\
\text { limpias con un aprovechamiento eficaz de los } \\
\text { recursos. En este escenario se da preponderan- } \\
\text { cia a las soluciones de orden mundial encami- } \\
\text { nadas a la sostenibilidad económica, social y } \\
\text { medioambiental, así como a una mayor igual- } \\
\text { dad, pero en ausencia de iniciativas adicionales } \\
\text { en relación con el clima (IPCC, 2007) }\end{array}$ \\
\hline
\end{tabular}

Tabla 2. Características de los escenarios $A 1 B, A 2$ y $B 1$ de acuerdo al IPCC

Fuente: elaboración propia

\section{Resultados}

\section{Estimación de temperatura y precipitación bajo escenarios de cambio climático para la RFPBOB utilizando MarkSimGCM}

A continuación se describen los resultados y análisis de las variables climáticas futuras: temperatura media promedio anual, precipitación promedio anual y mensual, para la RFPBOB. Los escenarios analizados en este trabajo son el A1B, A2 y B1, se analizan datos para cada una de las estaciones (tabla 1), los datos por cada estación son comparados frente a un valor de referencia o línea base simulado por MarkSimGCM para el periodo 1960-1990. 


\section{Escenario $A 1 B$}

A continuación se describen y analizan los resultados obtenidos en MarkSimGCM para temperatura y precipitación en el escenario A1B.

\section{- Temperatura}

En la tabla 3 se presentan los resultados estimados para cada una de las coordenadas señaladas. La diferencia entre el periodo de referencia respecto al año 2095 refleja una diferencia de $3,6^{\circ} \mathrm{C}$ equivalente al máximo incremento de temperatura en este escenario para la RFPBOB al final del siglo XIX. Los coeficientes de variación se encuentran entre
12,74 y $14,48 \%$ para los años simulados, mientras que para el periodo de referencia es de $17.37 \%$.

- Isotermas para la RFPBOB bajo el escenario A1B

Las isotermas obtenidas para el escenario A1B en los años 2040, 2070 y 2095, respectivamente, presentan una tendencia de aumento progresivo entre si, la orientación del incremento tiene dirección occidenteoriente. En todos los casos, las mayores temperaturas se encuentran al oeste, mientras que al este se hallan las más bajas (figuras 1,2 y 3 ), lo anterior puede ser a causa de fenómenos como la diferencia altitudinal dentro y fuera de la RFPBOB.

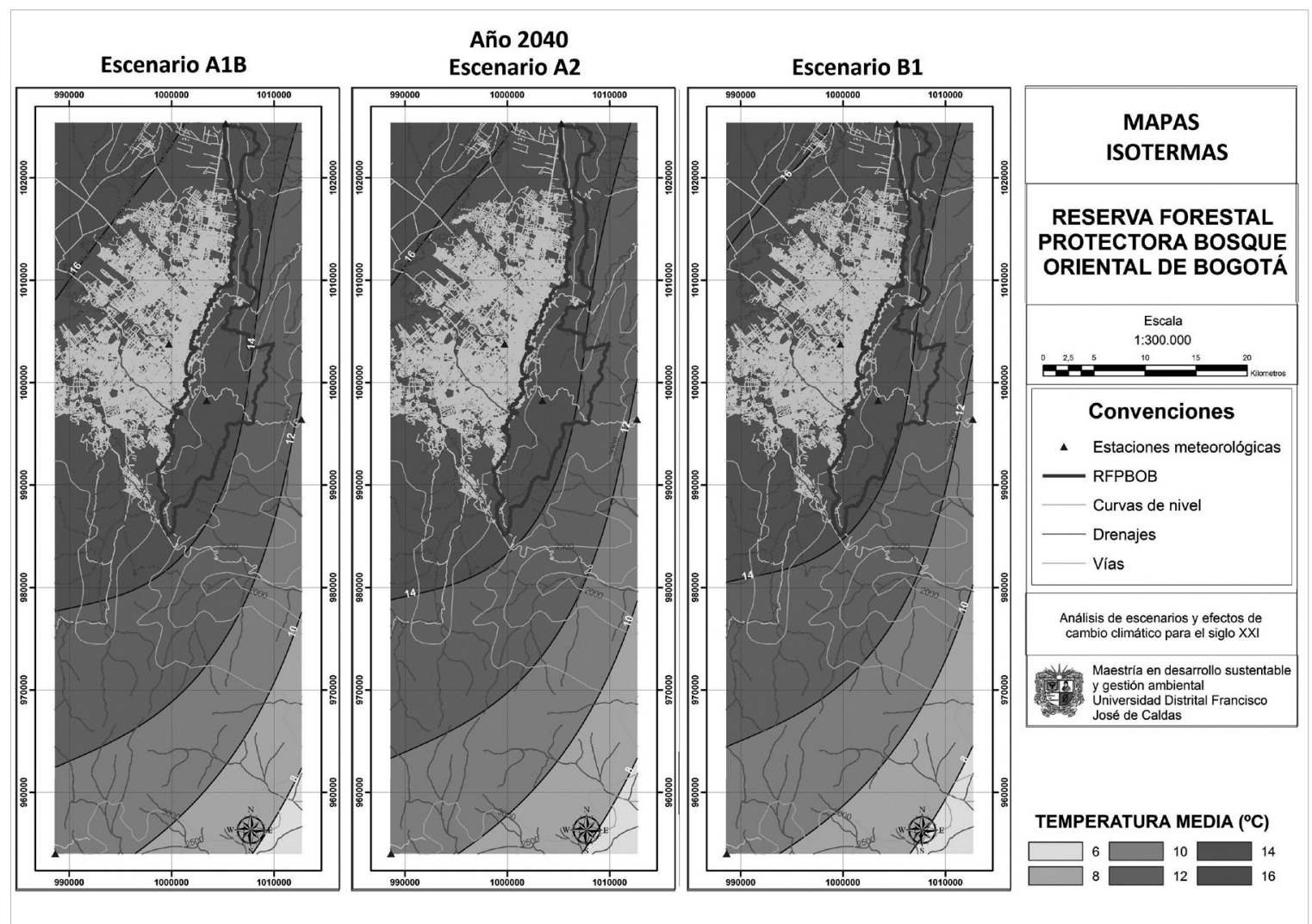

Figura 1. Variación de la temperatura escenarios A1B, A2 y B1 (año 2040) para la RFPBOB

Fuente: elaboración propia 


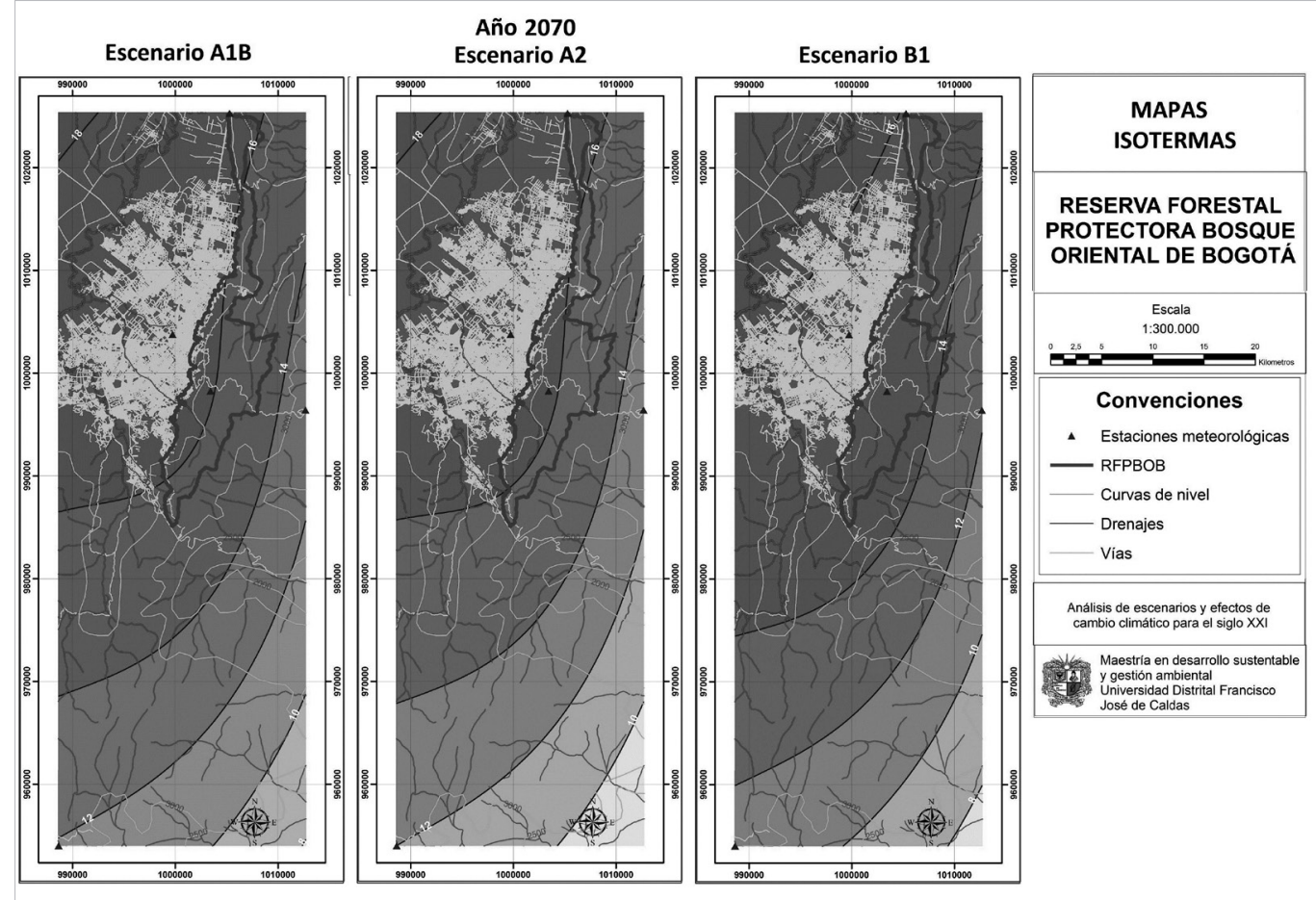

Figura 2. Variación de la temperatura escenarios A1B, A2 y B1 (año 2070) para la RFPBOB

Fuente: elaboración propia

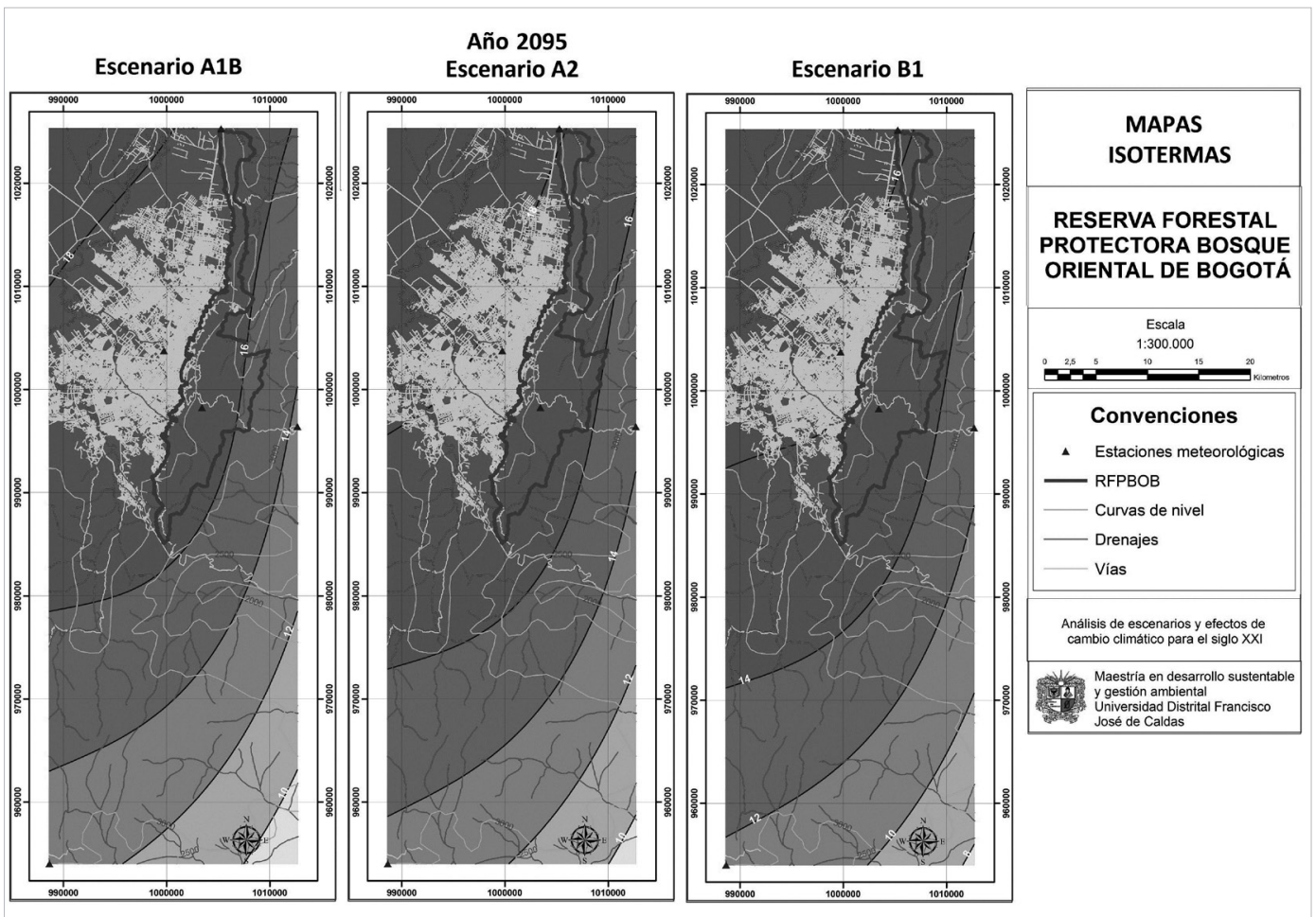

Figura 3. Variación de la temperatura escenarios $A 1 B, A 2$ y B1 (año 2095) para la RFPBOB

Fuente: elaboración propia 


\section{- Precipitación}

En la tabla 4, se presentan los valores correspondientes a precipitación anual y mensual promedio por estación y año analizado. En todas las estaciones, excepto en la Universidad Nacional, la tendencia es el incremento continuo en los tres años estudiados, lo que señala un aumento en el régimen de lluvias en el escenario A1B para la RFPBOB. Los datos reflejan un coeficiente de variación entre $21,10 \%$ y $19,44 \%$.

- Isoyetas para la RFPBOB bajo el escenario A1B

Las isoyetas modeladas para el escenario A1B en los años 2040, 2070 y 2095 presentan una tendencia de transición hacia el aumento en los tres años analizados (figuras 4, 5 y 6). Inicialmente para el año 2040, hay dos regiones claramente definidas con diferentes regímenes de precipitación. Al sur se presenta una zona de menor precipitación, el monto oscila entre 600 y $800 \mathrm{~mm}$, mientras que al centro y norte las lluvias son mayores con valores entre 800 y $1000 \mathrm{~mm}$.

Para el año 2070 se observan diferencias con respecto al año 2040. Se presenta un solo régimen de precipitación en el rango de 800 a $1000 \mathrm{~mm}$ de precipitación anual. Para el año 2095 en el escenario A1B se distinguen dos zonas; una oriental de la reserva que presenta precipitaciones entre 1000 y 1200 mm, y la occidental, más cercana a la ciudad con lluvias anuales entre 800 y $1000 \mathrm{~mm}$. Al revisar la tendencia de la precipitación en los tres años analizados, en este escenario se encuentra un aumento en la precipitación para el último año, 2095.

\begin{tabular}{|c|c|c|c|c|c|c|c|c|c|}
\hline \multirow{3}{*}{ Estación } & \multirow{3}{*}{$\begin{array}{l}\text { Ubicación } \\
\text { RFPBOB }\end{array}$} & \multicolumn{8}{|c|}{ Precipitación promedio (mm) } \\
\hline & & \multicolumn{2}{|c|}{ Línea base (1975) } & \multicolumn{2}{|c|}{ Año 2040} & \multicolumn{2}{|c|}{ Año 2070} & \multicolumn{2}{|c|}{ Año 2095} \\
\hline & & Anual & Mensual & Anual & Mensual & Anual & Mensual & Anual & Mensual \\
\hline $\begin{array}{l}\text { Venado del } \\
\text { oro }\end{array}$ & Central & 806,70 & 67,23 & 778,1 & 64,84 & 819,30 & 68,28 & 987,50 & 82,29 \\
\hline Los Tanques & Sur & 1162,20 & 96,85 & 1258,2 & 104,85 & 1371,80 & 114,32 & 1600,40 & 133,37 \\
\hline La Bolsa & Oriente & 1193,90 & 99,49 & 1110,5 & 92,54 & 1113,20 & 92,77 & 1343,30 & 111,94 \\
\hline UN & Occidental & 830,70 & 69,23 & 911,2 & 75,93 & 963,50 & 80,29 & 910,80 & 75,90 \\
\hline UDCA & Norte & 752,60 & 62,72 & 785 & 65,42 & 884,60 & 73,72 & 905,40 & 75,45 \\
\hline \multicolumn{2}{|c|}{ Promedio RFPBOB } & 949,22 & 79,10 & 968,60 & 80,72 & 1030,48 & 85,87 & 1149,48 & 95,79 \\
\hline \multicolumn{2}{|c|}{ Desviación estándar (S) } & 188,81 & 15,73 & 188,31 & 15,69 & 196,83 & 16,40 & 277,01 & 23,08 \\
\hline \multicolumn{2}{|c|}{ Coeficiente de variación (CV) } & $19,89 \%$ & $19,89 \%$ & $19,44 \%$ & $19,44 \%$ & $19,10 \%$ & $19,10 \%$ & $24,10 \%$ & $24,10 \%$ \\
\hline
\end{tabular}

Tabla 4. Resultados estimados para precipitación anual y mensual promedio en el escenario A1B

Fuente: elaboración propia

- Consideraciones generales sobre el escenario A1B

El escenario A1B para la RFPBOB muestra incrementos en la precipitación y temperatura respecto al clima actual. Para la temperatura media la estación más calurosa al año 2095 es la norte (UDCA) con $17,42^{\circ} \mathrm{C}$ y la más fría Los Tanques al sur con una temperatura media anual promedio de $12,68^{\circ} \mathrm{C}$. En relación con la precipitación, las mayores lluvias se encuentran al sur de la RFPBOB, seguidas de la parte oriental, mientras que en las regiones norte y oeste se observan regímenes de lluvias menores a la zona central y sur. En general el escenario A1B presenta cambios apreciables al compararse con las condiciones actuales de precipitación y temperatura tomadas del análisis climático realizado a partir de datos 
Ideam. En relación con las isoyetas en este escenario, desaparecen las tres regiones que se aprecian en la actualidad: norte, centro y sur, por franjas al sur y centro norte en 2040, una sola en 2070 y oriente, occidente en 2095. Las isoyetas para la $\mathrm{RFPBOB}$ en el escenario A1B muestran inicialmente disminución en la precipitación y posterior aumento frente a las condiciones actuales.

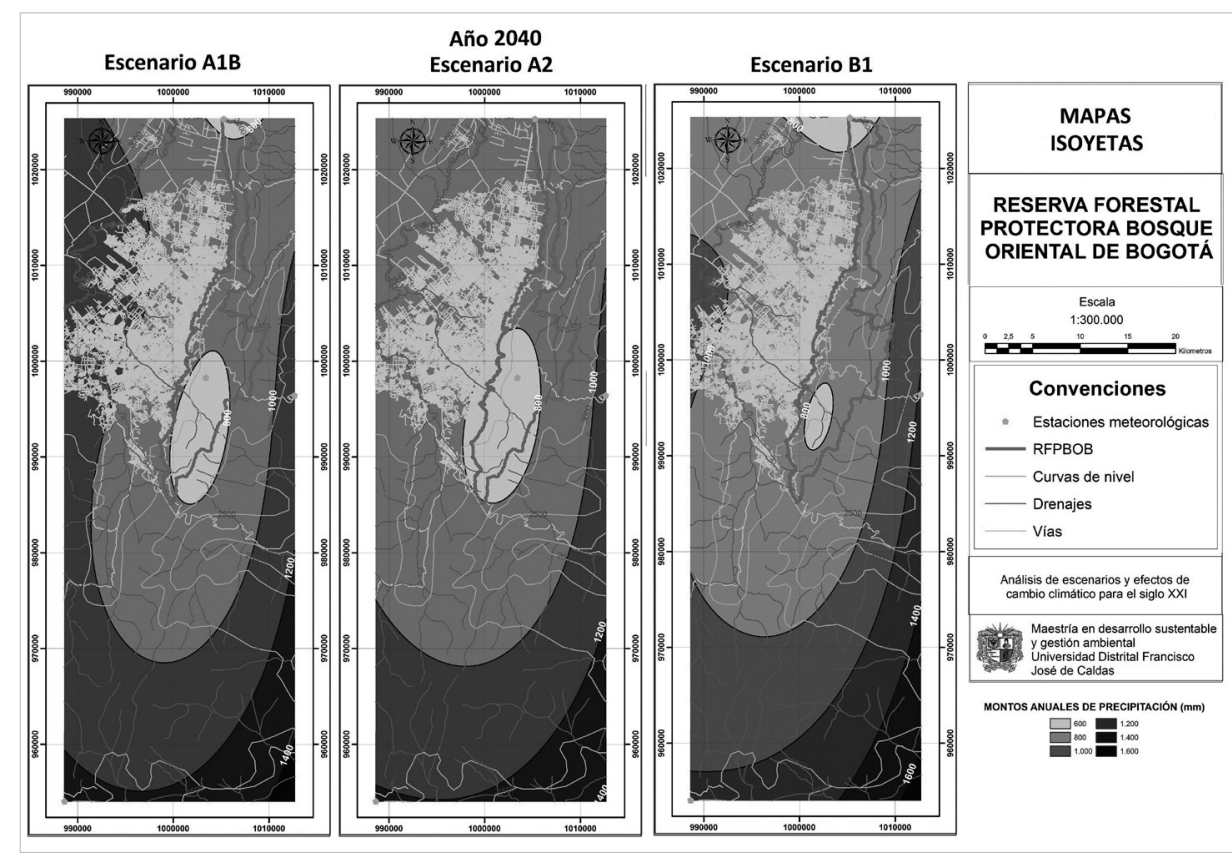

Figura 4. Variación de la precipitación escenarios A1B, A2 y B1 (año 2040) para la RFPBOB

Fuente: elaboración propia

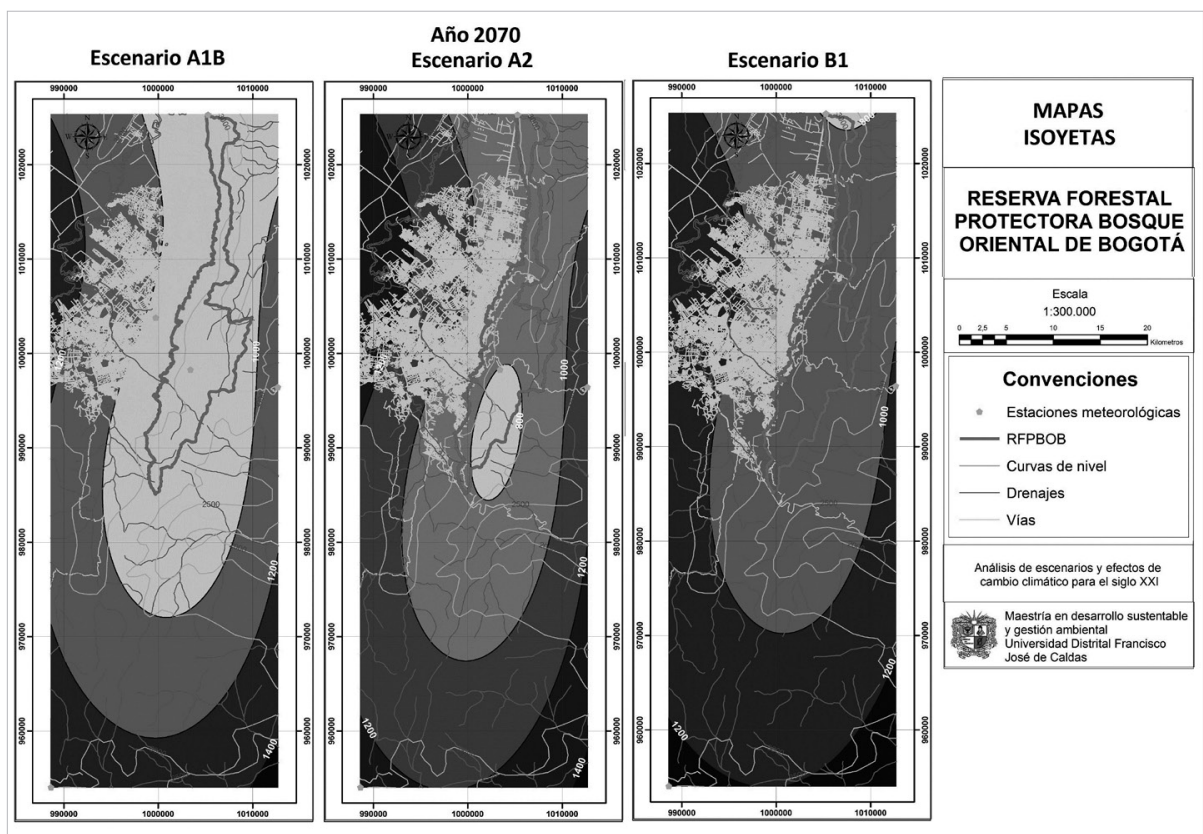

Figura 5. Variación de la precipitación escenarios A1B, A2 y B1 (año 2070) para la RFPBOB

Fuente: elaboración propia 


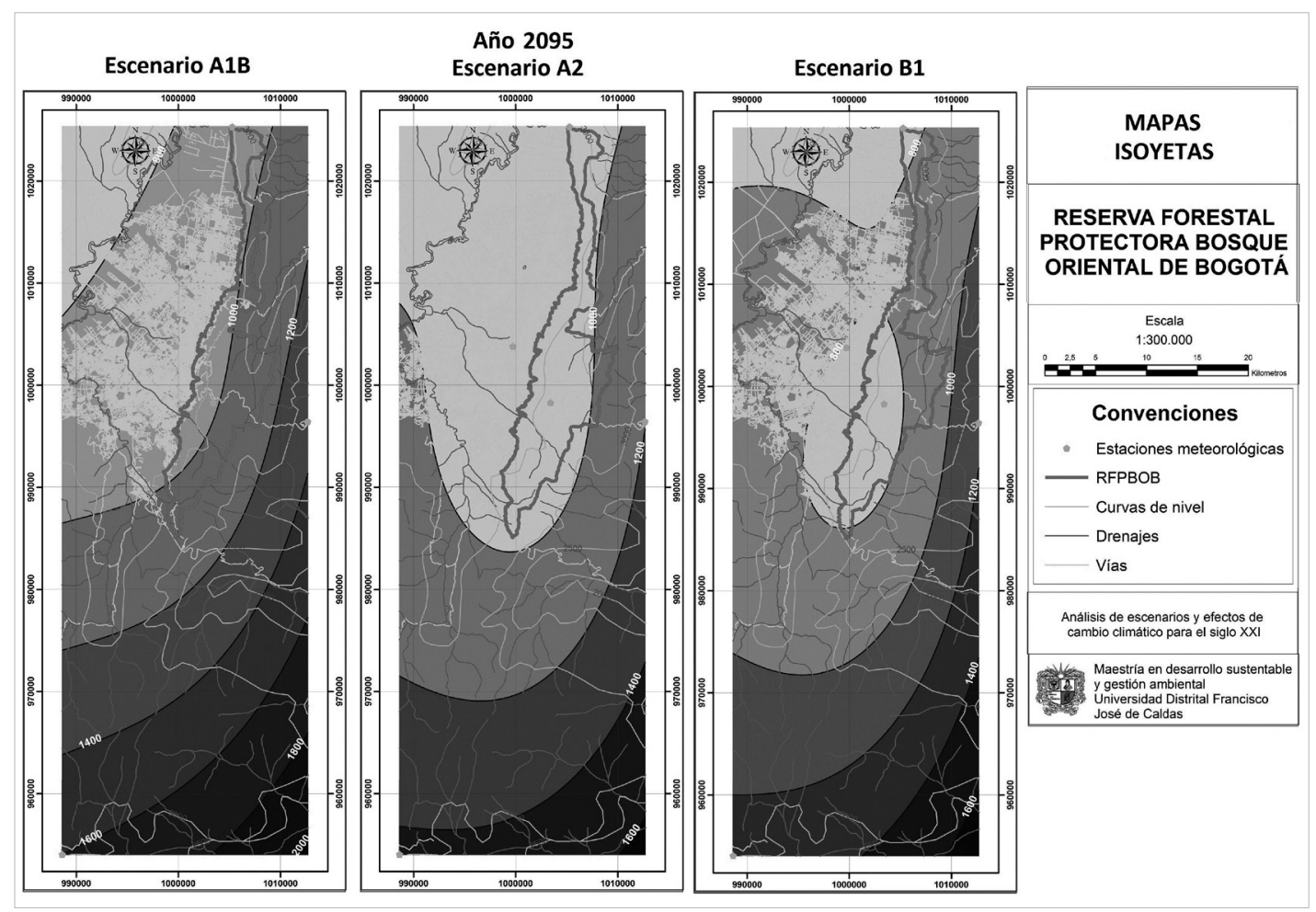

Figura 6. Variación de la precipitación escenarios A1B, A2 y B1 (año 2095) para la RFPBOB

Fuente: elaboración propia

\section{Escenario A2}

A continuación se describen y analizan los resultados obtenidos en MarkSimGCM para temperatura y precipitación en el escenario A2.

- Temperatura

En la tabla 5, se encuentran los resultados encontrados para cada una de las coordenadas estudiadas. En promedio para todas las estaciones se registra un incremento a través de los años. El cambio de temperatura media anual desde el periodo de referencia al año 2095 es de $4,3^{\circ} \mathrm{C}$, valor que muestra el incremento esperado para este escenario al final del siglo XIX. Respecto al escenario A1B, este es $0,7^{\circ} \mathrm{C}$ más caluroso, los coeficientes de variación para los años analizados están entre 12,29\% y 14,70\%.

- Isotermas para la RFPBOB bajo el escenario A2
Las isotermas obtenidas para el escenario A2 en los años 2040, 2070 y 2095 presentan una tendencia de aumento progresivo entre sí, la orientación del incremento tiene dirección occidente oriente, tal como se observó en el escenario A1B. En todos los casos, las mayores temperaturas se encuentran al oeste, mientras que al este se hallan las más bajas. $\mathrm{Al}$ año 2040 el promedio de la temperatura media se encuentra entre 14 y $16{ }^{\circ} \mathrm{C}$ en gran parte de la RFPBOB; sin embargo, en la porción más oriental, al centro de la reserva la temperatura está en el rango de $12 \mathrm{a} 14^{\circ} \mathrm{C}$. En 2070 la situación cambia, se distinguen dos franjas que dividen la reserva, una al oriente con temperatura media entre 14 y $16^{\circ} \mathrm{C}$ y una al occidente con un rango entre 16 y $18^{\circ} \mathrm{C}$, el comportamiento es similar al encontrado para este mismo año en el escenario A1B. Al 2095 la temperatura media promedio en toda la reserva está en el rango de 16 a $18^{\circ} \mathrm{C}$, situación que es homóloga a lo encontrado en el escenario A1B. 


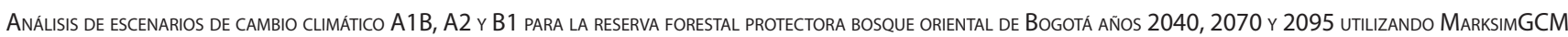

\begin{tabular}{|l|l|l|l|l|l|}
\hline \multirow{2}{*}{ Estación } & \multirow{2}{*}{ Ubicación } & \multicolumn{4}{|l|}{ Temperatura media $\left({ }^{\circ} \mathbf{C}\right)$} \\
\cline { 3 - 6 } & & Línea base (1975) & Año 2040 & Año 2070 & Año $\mathbf{2 0 9 5}$ \\
\hline Venado del oro & Central & 13,23 & 14,82 & 16,17 & 17,52 \\
\hline Los Tanques & Sur & 9,22 & 10,63 & 12,00 & 13,32 \\
\hline La Bolsa & Oriente & 9,57 & 11,69 & 13,02 & 14,36 \\
\hline UN & Occidental & 13,62 & 15,21 & 16,53 & 17,90 \\
\hline UDCA & Norte & 13,93 & 15,41 & 16,73 & 18,11 \\
\hline Promedio RFPBOB & & $\mathbf{1 1 , 9 2}$ & $\mathbf{1 3 , 5 5}$ & $\mathbf{1 4 , 8 9}$ & $\mathbf{1 6 , 2 4}$ \\
\hline Desviación estándar (S) & & 2,07 & 1,99 & 1,98 & 2,00 \\
\hline Coeficiente de variación (CV) & $17,37 \%$ & $14,70 \%$ & $13,28 \%$ & $12,29 \%$ \\
\hline
\end{tabular}

\section{Tabla 5.Resultados estimados para temperatura media en el escenario A2}

Fuente: elaboración propia

- Precipitación

En la tabla 6 se observan por separado los valores correspondientes a precipitación anual y mensual promedio por estación y año analizado. En todas las estaciones, excepto en la Universidad Nacional, la tendencia es de incremento continuo en los tres años estudiados, esto refleja un aumento en el régimen de lluvias en el escenario A2 para la RFPBOB. El coeficiente de variación para los años analizados en este escenario está en el rango de $17,69 \%$ a $18,77 \%$.

\begin{tabular}{|c|c|c|c|c|c|c|c|c|c|}
\hline \multirow{3}{*}{ Estación } & \multirow{3}{*}{$\begin{array}{l}\text { Ubicación } \\
\text { RFPBOB }\end{array}$} & \multicolumn{8}{|c|}{ Precipitación promedio (mm) } \\
\hline & & \multicolumn{2}{|c|}{ Línea base (1975) } & \multicolumn{2}{|c|}{ Año 2040} & \multicolumn{2}{|c|}{ Año 2070} & \multicolumn{2}{|l|}{ Año 2095} \\
\hline & & Anual & Mensual & Anual & Mensual & Anual & Mensual & Anual & Mensual \\
\hline Venado del oro & Central & 806,70 & 67,23 & 769,1 & 64,09 & 796,80 & 66,40 & 922,80 & 76,90 \\
\hline Los Tanques & Sur & 1162,20 & 96,85 & 1220,4 & 101,70 & 1269,70 & 105,81 & 1439,80 & 119,98 \\
\hline La Bolsa & Oriente & 1193,90 & 99,49 & 1052,7 & 87,73 & 1132,90 & 94,41 & 1195,80 & 99,65 \\
\hline UN & Occidental & 830,70 & 69,23 & 833,3 & 69,44 & 974,30 & 81,19 & 940,30 & 78,36 \\
\hline UDCA & Norte & 752,60 & 62,72 & 834,9 & 69,58 & 841,60 & 70,13 & 934,80 & 77,90 \\
\hline \multicolumn{2}{|c|}{ Promedio RFPBOB } & 949,22 & 79,10 & 942,08 & 78,51 & 1003,06 & 83,59 & 1086,70 & 90,56 \\
\hline \multicolumn{2}{|c|}{ Desviación estándar (S) } & 188,81 & 15,73 & 169,08 & 14,09 & 177,42 & 14,79 & 203,94 & 16,99 \\
\hline \multicolumn{2}{|c|}{ Coeficiente de variación (CV) } & $19,89 \%$ & $19,89 \%$ & $17,95 \%$ & $17,95 \%$ & $17,69 \%$ & $17,69 \%$ & $18,77 \%$ & $18,77 \%$ \\
\hline
\end{tabular}

Tabla 6. Resultados estimados para precipitación anual y mensual promedio en el escenario A2

Fuente: elaboración propia 
- Isoyetas para la RFPBOB bajo el escenario A2

Las isoyetas obtenidas para el escenario A2 en los años 2040, 2070 y 2095, respectivamente, presentan una tendencia de transición en los tres años analizados que va aumentando progresivamente. En el año 2040 se distinguen dos regiones con precipitaciones definidas. Al sur de la reserva el monto oscila entre 600-800 mm. Mientras que al centro y norte el monto anual de lluvias es mayor, con valores entre 800-1000 $\mathrm{mm}$, este comportamiento es similar al observado en el escenario A1B. Al año 2070, se muestra un corrimiento de las precipitaciones entre 600-800 $\mathrm{mm}$ hacia el sur oriente de la reserva. El resto de la RFPBOB continúa manifestando lluvias entre 800$1000 \mathrm{~mm}$. Para el año 2095 el panorama cambia en el escenario A2, la mayor parte de la reserva evidencia precipitaciones en un rango entre 800 y $1000 \mathrm{~mm}$. Se distinguen dos zonas entre las franjas oriente y occidente. La zona más oriental de la reserva presenta precipitaciones entre 1000 y $1200 \mathrm{~mm}$.

- Consideraciones generales sobre el escenario A2

Para el escenario A2 se muestran aumentos en la precipitación y temperaturas en las ubicaciones geográficas analizadas respecto al clima actual. Para la temperatura media anual la estación más calurosa al año 2095 es la UDCA al norte de la RFPBOB con $18.11^{\circ} \mathrm{C}$, mientras que al sur en la estación Los Tanques se encuentran las temperaturas más bajas. En relación con la precipitación los mayores montos se observan al sur de la RFPBOB, seguidas de la parte oriental. Al comparar las tendencias del escenario A2 con el escenario A1B se encuentran similitudes en el comportamiento de los máximos y mínimos de precipitación y temperatura media. Queda claro que el escenario A2 es mucho más caluroso que el A1B con aumentos de temperatura superiores en todas las estaciones analizadas. La precipitación se comporta de forma similar; sin embargo, en las estaciones central (Venado de Oro), sur (Los Tanques) y este (La Bolsa) los montos son menores para el año 2095 que los calculados en el escenario A1B. Las estaciones oeste y norte tienen mayores precipitaciones en el escenario A2 que las observadas en el A1B.

\section{Escenario B1}

A continuación se describen y analizan los resultados obtenidos en MarkSimGCM para temperatura, precipitación en el escenario B1.

\section{- Temperatura}

En la tabla 7 se encuentran los resultados encontrados para cada una de las coordenadas estudiadas. Al revisar los promedios y compararlos frente al valor del periodo de referencia, el incremento máximo se encuentra en el año 2095 , con un aumento de $2,5^{\circ} \mathrm{C}$, siendo este el incremento máximo para este escenario al final del siglo XIX. Al comparar este valor con el encontrado en los escenarios A1B y A2 se encuentra que este escenario presenta el menor incremento de temperatura. Los coeficientes de variación de los datos por año están entre 13,42 \% y 14,84 \%.

\begin{tabular}{|l|l|l|l|l|l|}
\hline \multirow{2}{*}{ Estación } & \multirow{2}{*}{ Ubicación } & \multicolumn{2}{|l|}{ Temperatura media $\left({ }^{\circ} \mathbf{C}\right)$} \\
\cline { 3 - 6 } & & Línea base (1975) & Año 2040 & Año 2070 & Año 2095 \\
\hline Venado del oro & Central & 13,23 & 14,64 & 15,33 & 15,68 \\
\hline Los Tanques & Sur & 9,22 & 10,48 & 11,13 & 11,61 \\
\hline La Bolsa & Oriente & 9,57 & 11,51 & 12,18 & 12,62 \\
\hline UN & Occidental & 13,62 & 15,02 & 15,71 & 16,06 \\
\hline UDCA & Norte & 13,93 & 15,26 & 15,90 & 16,26 \\
\hline Promedio RFPBOB & & $\mathbf{1 1 , 9 2}$ & $\mathbf{1 3 , 3 8}$ & $\mathbf{1 4 , 0 5}$ & $\mathbf{1 4 , 4 5}$ \\
\hline Desviación estándar (S) & & 2,07 & 1,99 & 1,99 & 1,94 \\
\hline Coeficiente de variación (CV) & $17,37 \%$ & $14,84 \%$ & $14,18 \%$ & $13,42 \%$ \\
\hline
\end{tabular}

Tabla 7. Resultados estimados para temperatura media en el escenario B1 
- Isotermas para la RFPBOB bajo el escenario B1

Las isotermas obtenidas para el escenario B1 en los años 2040, 2070 y 2095 no presentan cambios considerables entre sí. El rango de temperatura predominante está entre 14 y $16^{\circ} \mathrm{C}$. En el año 2040 la temperatura media promedio para la reserva está mayoritariamente entre 14 y $16^{\circ} \mathrm{C}$, excepto para la porción más oriental de la RFPBOB donde la temperatura está en el rango de 12 a $14^{\circ} \mathrm{C}$, situación similar a la descrita en el escenario A2. Para el año 2070 se encuentra que la temperatura media para toda la reserva está en el rango de 14 a $16^{\circ} \mathrm{C}$, estos mismos valores se observaron para las isotermas de este año en el escenario A1B. Al 2095, la temperatura media permanece en el rango del año 2070 , esto es entre 14 y $16^{\circ} \mathrm{C}$.

\section{- Precipitación}

En la tabla 8, se observan por separado los valores correspondientes a precipitación anual y mensual promedio por estación y año analizado para el escenario B1. El comportamiento en la precipitación es variado en las estaciones al comparase los tres años estudiados y cada estación.
- Isoyetas para la RFPBOB bajo el escenario B1

Las isoyetas obtenidas para el escenario B1 a partir de los datos de la tabla 8 en los años 2040, 2070 y 2095 presentan variaciones apreciables en los tres años analizados. Las isoyetas del escenario B1 al año 2040 presentan tres regiones: una, la más pequeña, al extremo norte, otra al sur dentro de la reserva y una tercera, la más grande, que conforma el resto de la RFPBOB. Cada una de estas presenta montos de precipitación distintos.

En las porciones extremo norte y sur la precipitación se encuentra entre 600 y $800 \mathrm{~mm}$, para el resto de la reserva las lluvias están entre 800 y 1000 mm al año. Este comportamiento en las precipitaciones es similar a lo encontrado en los escenarios A1B y A2, particularmente en la distribución de los montos de lluvias. Para 2070, se observa un solo monto de precipitación a lo largo de la reserva entre 800 y 1000 $\mathrm{mm}$. En el año 2095 se distinguen dos grandes zonas, una al sur occidente y otra al centro y nororiente. En la primera, el rango de precipitación se encuentra entre 600 y $800 \mathrm{~mm}$, mientras que en la segunda la precipitación se sitúa entre 800 a $1000 \mathrm{~mm}$, lo cual presenta similitudes con el comportamiento visto en el escenario A2 en el año 2040.

\begin{tabular}{|c|c|c|c|c|c|c|c|c|c|}
\hline \multirow{3}{*}{ Estación } & \multirow{3}{*}{$\begin{array}{l}\text { Ubicación } \\
\text { RFPBOB }\end{array}$} & \multicolumn{8}{|c|}{ Precipitación promedio (mm) } \\
\hline & & \multicolumn{2}{|c|}{ Línea base (1975) } & \multicolumn{2}{|c|}{ Año 2040} & \multicolumn{2}{|c|}{ Año 2070} & \multicolumn{2}{|c|}{ Año 2095} \\
\hline & & Anual & Mensual & Anual & Mensual & Anual & Mensual & Anual & Mensual \\
\hline Venado del oro & Central & 806,70 & 67,23 & 805,2 & 67,10 & 817,30 & 68,11 & 761,60 & 63,47 \\
\hline Los Tanques & Sur & 1162,20 & 96,85 & 1240,2 & 103,35 & 1262,60 & 105,22 & 1299,00 & 108,25 \\
\hline La Bolsa & Oriente & 1193,90 & 99,49 & 1217 & 101,42 & 1024,60 & 85,38 & 1166,80 & 97,23 \\
\hline UN & Occidental & 830,70 & 69,23 & 885,1 & 73,76 & 919,90 & 76,66 & 794,80 & 66,23 \\
\hline UDCA & Norte & 752,60 & 62,72 & 766,2 & 63,85 & 795,70 & 66,31 & 768,80 & 64,07 \\
\hline \multicolumn{2}{|c|}{ Promedio RFPBOB } & 949,22 & 79,10 & 982,74 & 81,90 & 964,02 & 80,34 & 958,20 & 79,85 \\
\hline \multicolumn{2}{|c|}{ Desviación estándar (S) } & 188,81 & 15,73 & 204,50 & 17,04 & 170,07 & 14,17 & 228,42 & 19,04 \\
\hline \multicolumn{2}{|c|}{ Coeficiente de variación (CV) } & $19,89 \%$ & $19,89 \%$ & $20,81 \%$ & $20,81 \%$ & $17,64 \%$ & $17,64 \%$ & $23,84 \%$ & $23,84 \%$ \\
\hline
\end{tabular}

Tabla 8. Resultados estimados para precipitación anual y mensual promedio en el escenario B1

Fuente: elaboración propia

- Consideraciones generales sobre el escenario B1

De los resultados del escenario B1 se puede concluir un panorama de aumento en la temperatura y precipitación respecto al clima actual, pero en menor medida que lo observado en los escenarios A2 y A1B. Para la temperatura media la estación más calurosa al año 2095 es la norte, estación UDCA y la de menor aumento Los Tanques al sur, como se encontró en los escenarios A2 y A1B. Al 
revisar la precipitación, los mayores montos de lluvias se registran al sur oriente de la RFPBOB en las estaciones Los Tanques y La Bolsa. Las secciones central y occidental muestran precipitaciones menores a las observadas en la zona suroriental, pero superiores a las de la sección norte de la RFPBOB, donde se observan las menores precipitaciones promedio anuales. Al comparar las tendencias del escenario B1, con lo observado en los escenarios A1B y A2 se encuentran similitudes en el comportamiento de los máximos y mínimos de precipitación y temperatura media; sin embargo, se encuentra que el escenario B1 es el menos caluroso de los tres escenarios analizados.

\section{Consideraciones finales}

Se estableció el comportamiento de las variables climáticas temperatura y precipitación de la Reserva Forestal Protectora Bosque Oriental de Bogotá, bajo los escenarios de cambio climático A1B, A2 y B1 usando el simulador MarkSimGCM que se soporta a su vez en los modelos de circulación general: CNRM-CM3, CSIRO-Mk3.5, ECHam5 y MIROC3.2 y en datos de la base climática WorldClim. Los años estudiados fueron: 2040, 2070 y 2095, años que corresponden con periodos analizados en escenarios de cambio climático para Colombia en estudios previos realizados particularmente por el Ideam. El área de estudio se observa en el mapa de la figura 7.

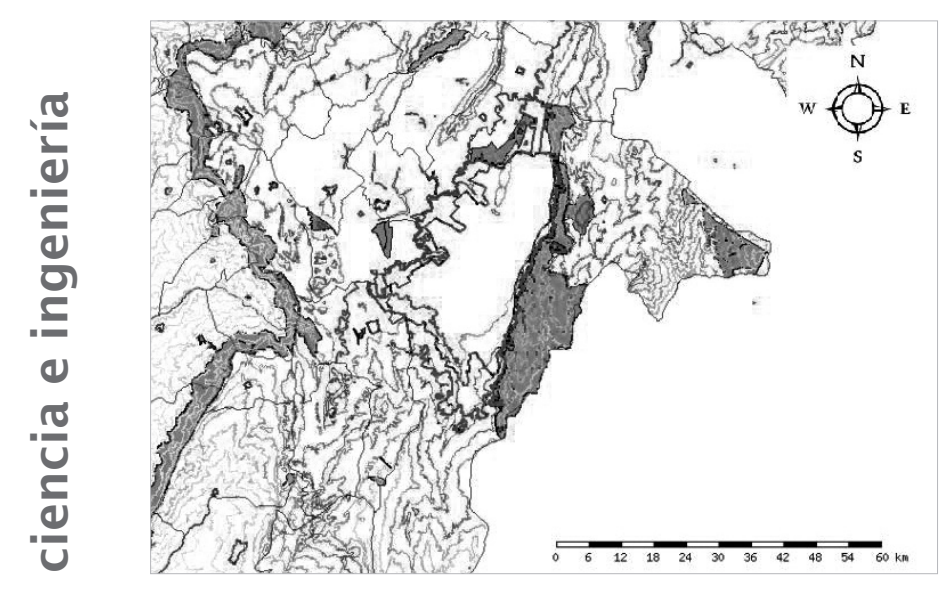

Figura 7. Reserva forestal protectora Cerros Orientales de Bogotá

Fuente: Corporación autónoma regional de Cundinamarca (2012)
Para el escenario A1B se encontró que la temperatura media en el año 2040 estará en gran parte de la reserva entre 14 a $16^{\circ} \mathrm{C}$, mientras que para la sección central más oriental la temperatura estará en el rango de $12 \mathrm{a} 14^{\circ} \mathrm{C}$. Para 2070 la mayor parte de la reserva continuaría presentando una temperatura media anual entre 14 a $16^{\circ} \mathrm{C}$; sin embargo, al occidente aparece una zona que presenta mayor temperatura, entre 16 y $18^{\circ} \mathrm{C}$. En este año se diferencian dos regiones, una oriental con menor temperatura y una occidental más cálida. Al año 2095 la temperatura media promedio anual se incrementa en comparación con los años 2040 y 2070. Se observó para este año que la mayor parte de la reserva presenta una temperatura entre 16 y $18^{\circ} \mathrm{C}$. En la parte más oriental al centro se destaca una pequeña zona que mantiene la temperatura del año 2070 entre 14 y $16^{\circ} \mathrm{C}$. La estación más calurosa al año 2095 fue la norte (UDCA) con $17,42^{\circ} \mathrm{C}$ y la más fría Los Tanques al sur con una temperatura media anual promedio de $12,68{ }^{\circ} \mathrm{C}$. En relación con la precipitación en este escenario, las mayores lluvias se encontraron al sur de la RFPBOB, seguidas de la parte oriental, mientras que en las regiones norte y oeste se observaron regímenes de lluvias menores a la zona central y sur. Inicialmente para el año 2040, se observaron dos regiones claramente definidas con diferentes regímenes de precipitación. $\mathrm{Al}$ sur se revela una zona de menor precipitación donde el monto oscila entre 600 y $800 \mathrm{~mm}$, mientras que al centro y norte las lluvias son mayores con valores entre 800 y $1000 \mathrm{~mm}$. En 2070 se percibe una situación que varía considerablemente respecto al año 2040. Se encontró un solo régimen de precipitación para la RFPBOB que está en el rango de 800 a $1000 \mathrm{~mm}$ de precipitación anual. Para el año 2095 se distinguen dos zonas entre las regiones oriente y occidente. La zona más oriental de la reserva y por ende la más alta presentó precipitaciones entre 1000 y 1200 mm, mientras que la occidental, más cercana a la ciudad mostró lluvias anuales entre 800 y 1000 mm. En general el escenario A1B presentó cambios apreciables al compararse con las condiciones actuales de precipitación y temperatura tomadas del análisis climático realizado a partir de datos de Ruiz (2010) y (2011).

Para el escenario A2 se encontraron aumentos en la precipitación y temperatura en las ubicaciones 
geográficas analizadas para la $\mathrm{RFPBOB}$ respecto al clima actual. Al año 2040 el promedio de la temperatura media se determinó entre 14 y $16^{\circ} \mathrm{C}$ en gran parte de la RFPBOB; sin embargo, en la porción más oriental, al centro de la reserva la temperatura se observó en el rango de 12 a $14^{\circ} \mathrm{C}$. En 2070 la situación cambia, se distinguen dos franjas que dividieron la reserva, una al oriente con temperatura media entre 14 y $16^{\circ} \mathrm{C}$ y una al occidente con un rango entre 16 y $18^{\circ} \mathrm{C}$. $\mathrm{Al}$ 2095 la temperatura media promedio en toda la reserva se ubicó en el rango de 16 a $18^{\circ} \mathrm{C}$. Para los años 2070 y 2095 el comportamiento mostró el mismo rango de incremento del escenario A1B para estos mismos años. La estación más calurosa al año 2095 fue la UDCA al norte de la $\mathrm{RFPBOB}$ con $18,11^{\circ} \mathrm{C}$, mientras que al sur en la estación Los Tanques se encontraron las temperaturas más bajas. En relación con la precipitación, los montos más grandes se observaron al sur de la reserva seguidas de la parte oriental. En el año 2040 se distinguieron dos regiones con precipitaciones definidas. Al sur de la reserva el monto oscilóentre $600-800 \mathrm{~mm}$, mientras que al centro y norte el monto anual de lluvias es mayor con valores entre $800-1000 \mathrm{~mm}$, este comportamiento fue similar al observado en el escenario A1B. Al 2070, se observó un desplazamiento de las precipitaciones entre 600-800 $\mathrm{mm}$ hacia el sur oriente de la reserva. El resto de la RFPBOB continúo manifestando lluvias entre 800 y $1000 \mathrm{~mm}$. Para 2095 el panorama cambio en el escenario A2, la mayor parte de la reserva mostró precipitaciones en un rango entre 800 y $1000 \mathrm{~mm}$. Se encontraron dos zonas entre las franjas oriente y occidente. La zona más oriental de la reserva evidenció precipitaciones entre 1000-1200 mm.

$\mathrm{Al}$ comparar las tendencias del escenario A2 con el escenario A1B se encontraron similitudes en el comportamiento de los máximos y mínimos de precipitación y temperatura media. El escenario A2 es mucho más caluroso que el A1B con aumentos de temperatura superiores en todas las estaciones analizadas. Por otro lado, la precipitación se comportó de forma similar; sin embargo, en las estaciones central (Venado de Oro), sur (Los Tanques) y este (La Bolsa) las precipitaciones fueron menores para el año 2095 que las calculadas en el escenario
A1B. Las estaciones oeste y norte tuvieron mayores precipitaciones en el escenario A2 que las observadas en el A1B.

En el escenario B1 se espera un panorama de aumento en la temperatura y precipitación respecto al clima actual, pero en menor medida que lo observado en los escenarios A2 y A1B. En el año 2040 la temperatura media promedio para la reserva estuvo mayoritariamente entre 14 y $16^{\circ} \mathrm{C}$, excepto para la porción más oriental de la RFPBOB donde la temperatura se ubicó en el rango de 12 a $14^{\circ} \mathrm{C}$, situación semejante a la descrita en el escenario A2. Para 2070 y 2095 se encontró que la temperatura media para toda la reserva esta en el rango de 14 a $16^{\circ} \mathrm{C}$. La estación más calurosa al año 2095 fue la norte, estación UDCA y la de menor aumento Los Tanques al sur, tal y como se encontró en los escenarios A2 y A1B. Al revisar la precipitación, los mayores montos de lluvias se registraron al sur oriente de la RFPBOB en las estaciones Los Tanques y La Bolsa. Las secciones central y occidental mostraron precipitaciones menores a las observadas en la zona suroriental, pero superiores a las de la sección norte de la RFPBOB, donde se encontraron las menores precipitaciones promedio anuales. $\mathrm{Al}$ año 2040 se observaron tres regiones con diferentes montos de precipitación: una, la más pequeña al extremo norte, otra al sur dentro de la reserva y una tercera la más grande que conforma el resto de la RFPBOB. En las porciones extremo norte y sur la precipitación se ubicóentre $600-800 \mathrm{~mm}$, para el resto de la reserva las lluvias estuvieron entre 800-1000 $\mathrm{mm}$ al año. Este comportamiento en las precipitaciones recordó en alguna forma lo observado en los escenarios A1B y A2, particularmente en la distribución de los montos de lluvias. Para 2070, se observó un solo régimen de precipitación a lo largo de la reserva entre 800 y $1000 \mathrm{~mm}$. Para el año 2095 se distinguieron dos grandes zonas, una al sur occidente y otra al centro y nororiente. En la primera el rango de precipitación se encontró entre 600-800 mm, mientras que en la segunda la precipitación se sitúo entre 800-1000 mm, lo cual presentó similitudes con el comportamiento visto en el escenario A2 en el año 2040. Al comparar las tendencias del escenario $\mathrm{B} 1$, con lo observado en los escenarios A1B y A2 
se encontraron similitudes en el comportamiento de los máximos y mínimos de precipitación y temperatura media discutidos; sin embargo, se observó que el escenario B1 es el menos caluroso de los tres analizados.

Los resultados de las tendencias en la precipitación y temperatura indican cambios, particularmente de aumento en la temperatura, y en menor medida en la precipitación. Es importante considerar que, a pesar de que las estimaciones obtenidas en este trabajo concuerdan de alguna forma con otros estudios realizados, en este momento no es posible determinar con exactitud las condiciones climáticas futuras no importando el modelo que se utilice (Wilches, 2008). No obstante, a través de ejercicios como este se puede anticipar de alguna forma los cambios en variables climáticas relevantes, aportando información de apoyo a los procesos de toma de decisiones y en general en el manejo sustentable de la RFPBOB.

\section{Agradecimientos}

Los autores desean expresar sus agradecimientos a los Doctores: Celso Libardo Mateus Pineda y Roberto Emilio González Cubillos, por sus importantes consideraciones en la revisión y evaluación de la investigación. A Cesar Augusto Ruiz Agudelo, Biólogo, coordinador de servicios ecosistémicos de conservación internacional, por su apoyo e información relevante que fue utilizada y tomada como referencia en este trabajo. A Diego Beltrán, Ingeniero Forestal, por su valioso apoyo en el manejo de sistemas de información geográfica y el tratamiento de datos para la realización del análisis climático actual de la RFPBOB.

\section{Referencias bibliográficas}

Andrade, G., Mesa, C., Ramirez, A. y Remolina, F. (2008). Estructura ecológica principal y áreas protegidas de Bogotá. Foro nacional ambiental, documento de políticas públicas, 2-3.

Batchelet, D., Neilson, R., Lenihan, J. and Drapek, R. (2001). Climate change effects on vegetation distribution and carbon budget in the United States,Ecosystems, 4, 164-185.
Bernier, P. y Shoene, D. (2009). La adaptación de los bosques y su ordenación al cambio climático: una visión de conjunto. Unasylva, 60, (231-232).

Carrizosa, J. (2007). La sabana de Bogotá y los ecosistemas relacionados en el 2007. Bogotá: FESCOL.

Castro, M., Martín Vide, J. y Alonso, S. (2005). El clima de españa: pasado, presente y escenarios de clima par el siglo XXI. En J. Moreno, Evaluación preliminar de los impactos en España por efecto del cambio climático. Madrid: Ministerio de Ambiente.

Chapin, F., Zaveleta, E., Eviner, V., Naylor, R., Vitousek, S. and Lavorel, M. (2000). Consequences of changing biotic diversity. Nature, 405(234-242).

Corporación autónoma regional de Cundinamarca (CAR) (2013). Línea Base ambiental CAR Cartografía en Línea. Obtenido de CAR Cundinamarca. Recuperado de http://www.car. gov.co/tools/marco.php?idcategoria $=1267$

Cortes, S., Van der Hammen, T. y Rangel, O. (1999). Comunidades vegetales y patrones de degradación y sucesión en la vegatación de los cerros occidentales de Chia Cundinamarca-Colombia. Revista de la academia colombiana de ciencias, XXIII (89), 529-554.

Dobbs, C., Francisco, J. and Wayne, C. (2011). A framework for developing urban forest ecosystem services and goods indicators. Landscape and urban planning, (99), 96-206.

Groot, R. and Wilson, M. (2002). A typology for the classification, description and valuation of ecosystem functions, good and services. Ecological economics, 41(3), 393-408.

Guerrero, O. (agosto de 2006). Una mirada hacia los cerros orientales de Bogotá. Recuperado de http://www.premioreportagem.org.br/article. sub? docId $=23375 \& c=$ Colômbia\&cRef $=$ Colom bia\&year $=2007 \&$ date $=$ agosto 2006

Hijmans, R., Cameron, S., Parra, J., Jones, P. and Jarvis, A. (2005). Very high resolution interpolated climate surface for global land areas. International Journal of Climatology, 25, 1965-1978.

Instituto Colombiano de Estudios Ambientales (IDEAM). (2010). Estrategia nacional de 
educación, formación y sensibilización de públicos sobre cambio climático. Bogotá: Ideam.

Intergovernmental Panel on Climate Change (IPCC). (2001). Climate change 2001. The scientific basis. Cambridge: Cambridge university press.

Intergovernmental Panel on Climate Change (IPCC.) (2007). General guidelines on the use of scenario data for climate impact and adaptation assessment. Helsinki, Finland: WMO-UNEP.

Johns, T., Gregory, J. et al. (2003). Anthropogenic climate change for 1860 to 2100 simulated with the HadCM3 model under updated emissions scenarios. Climate Dynamics, 20,583-612.

Jones, P. and Thornton, P. (1993). A rainfall generator for agricultural applications in the tropics. Agricultural and forest meteorology, 63, 1-19.

Jones, P., and Thornton, P. (1993). A rainfall generator for agricultural applications in the tropics. Agricultural and forest meteorology, 63, 1-19.

Jones, P., Thornton, P. and Heinke, J. (2009). Generating characteristic daily weather data using downscaled climate model data from the IPCC's fourth assessment. Project Report. Nairobi, 1-24.

Magaña, V., Conde, C., Sanchez, O. y Gay, C. (2000). Evaluación de escenarios regionales de clima actual y de cambio climático futuro para México. En México: una visión hacia el siglo XXI. El cambio climático en México. Mexico.

Montes, C. Sala, O. (2007). La Evaluación de los Ecosistemas del Milenio. Las relaciones entre el funcionamiento de los ecosistemas y el bienestar humano. Revista Ecosistemas, 16(3).

Mahlman, J. (1997). Uncertainties in projections of humancaused climate warming. Science, 278, 1416-1417.

McKenzie, D., Peterson, D., Peterson, D., andThornton, P. (2003). Limatic and biophysical controls on conifer species distrinutions in mountain forest of Washington State, USA. Journal of Biogeography, 30, 1093-1108.
Millar, C., Stephenson, N. and Stephens, S. (2007). Climate change and forests of the future: Managing in the face of uncertainty. Ecological Applications, 17(8), 2145-2151.

Nitschke, C., and Innes, J. (2008). A tree and climate assessment tool for modelling ecosystem response to climate change. Ecological modelling, 210, 263-277.

Pabón, J. (2006). El cambio climático en Colombia. Bogotá: Universidad Nacional de Colombia.

Ruiz, J. (2011). Escenarios de cambio climático panorama 2011-2100. Bogotá: PRICC.

Ruiz, J. F. (2010). Cambio climático en temperatura, precipitación y humedad relativa para Colombia usando modelos mteorológicos de alta resolución (panorama 2011-2100). Bogotá: IDeam.

Sanchez. (2007). Reserva forestal protectora- cerros orientales de Bogotá. Bogotá: Personeria de Bogotá.

Turner, M. and Chapin III , F. (2005). Causes and consequences if spatial heterogeneity in ecosystem function. En G. Lovett, C. Jones, M. Turnes y K. Weathers. Ecosystem function in heterogeneous landscapes. New York: Springer-Verlag.

Watson, R., Zinyowera, M. and Moss, R. (1996). Impacts, adaptations and mitigation of climate change: scientific-technical analysis. Contribution of the working group II to the second assessment report of the intergovernmental panel on climate change. Cambridge: Cambridge University Press.

Wiesner, D. (2006). Formulación del plan zonal de ordenamiento y gestión del territorio para la franja de adecuación o transición entre la ciudad y los cerros orientales. Bogotá: Alcaldia Mayor de Bogotá.

Wilches, G. (2008). Programa Conjunto de Integración de ecosistemas y adaptación al cambio climático. Bogota. 